\title{
Plasmonic enhancement of High Harmonic Generation revisited: Predominance of Atomic Line Emission
}

\author{
M. Sivis ${ }^{1}$, M. Duwe ${ }^{1}$, B. Abel ${ }^{2}$, and C. Ropers ${ }^{1}$ \\ 1 Courant Research Center Nano-Spectroscopy and X-Ray Imaging, University of Göttingen, 37077 \\ Göttingen, Germany \\ 2 Ostwald-Institute for Physical and Theoretical Chemistry, University of Leipzig, 04103 Leipzig, \\ Germany
}

\begin{abstract}
We demonstrate nanostructure-enhanced extreme ultraviolet fluorescence from noble gases driven by low-energy, few-cycle light pulses. Despite sufficient local intensities, plasmon-enhanced high harmonic generation is not observed, which follows from the small, nanometer-size coherent source volume.
\end{abstract}

\section{Introduction}

Plasmonic nanostructures with tailored resonances can be utilized to control and enhance optical fields, which allows for the amplification of numerous linear and nonlinear optical processes [1-4].

Here we demonstrate nanostructure-enhanced atomic line emission (ALE) from noble gases driven by short nJ-pulses at high repetition rate. Recently, comparable experimental conditions were reported to cause intense high harmonic generation (HHG) in the extreme ultraviolet (EUV) [5]. In contrast, our study, involving different noble gases and nanostructure geometries, leads us to the conclusion that primarily incoherent fluorescence from neutral and ionized gas atoms can be e fficiently enhanced in plasmonic nanostructures, as opposed to HHG [6].

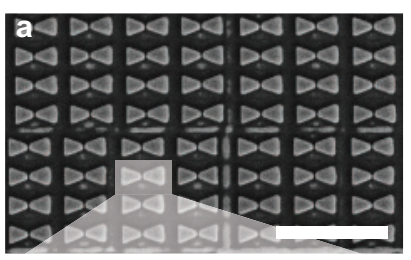

b

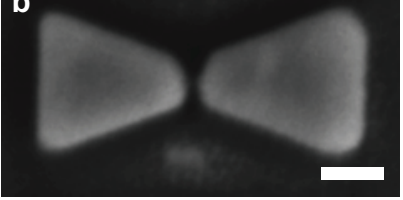

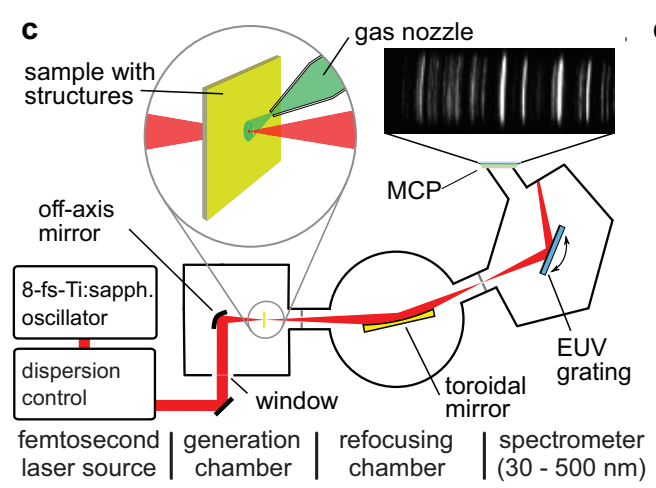

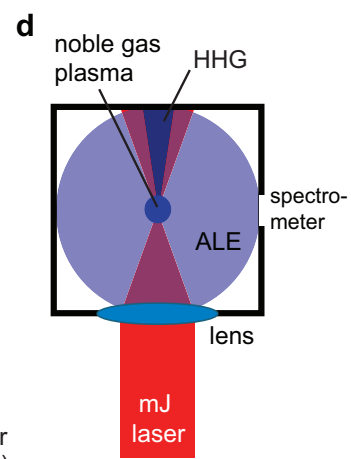

Fig. 1. a),b) Scanning electron micrograph of bow tie antennas (scale bars: a: $1 \mu \mathrm{m}$; b: 100 nm). The gold nanostructures are fabricated in arrays of $20 \times 20 \mu \mathrm{m}^{2}$ size on smooth sapphire substrates using focused ion beam etching. c) Schematic of the experimental setup. The inset shows an image of the phosphor screen recorded with the CCD camera. The image corresponds to the xenon measurement shown in Fig. 2b. d) Setup for reference measurements with amplified mJ-pulses (40 fs @ $1 \mathrm{kHz}$ rep. rate and $800 \mathrm{~nm}$ central wavelength) using the same spectrometer and gas nozzle as in c.

This is an Open Access article distributed under the terms of the Creative Commons Attribution License 2.0, which permits unrestricted use, distribution, and reproduction in any medium, provided the original work is properly cited. 


\section{Nanostructures and experimental setup}

Our study employs arrays of bow tie nanoantennas (cf. Fig. 1a) prepared by focused ion beam etching of smooth gold films (50-100 nm thickness, thermally evaporated) on dielectric sapphire substrates. The radii of tip curvature and the distance of the bow tie triangles are about $20 \mathrm{~nm}$ (Fig. 1b). The geometry of the bow ties is optimized for high field-enhancement and resulting nonlinear light emission.

The experimental setup is shown in Fig. 1c. A sample containing the nanostructures is mounted on micro-translation stages in a high vacuum chamber, and a moveable metallic nozzle (100 $\mu \mathrm{m}$ inner diameter) provides a gas jet to the structures (backing pressure 500 mbar). Dispersion controlled 8-fs light pulses from a $78 \mathrm{MHz}$ titanium:sapphire laser oscillator are focused to peak intensities exceeding $10^{11} \mathrm{Wcm}^{-2}$. Optical excitation is provided at normal incidence with a central wavelength of 800 $\mathrm{nm}$ and polarized along the bow tie main axis. The emitted radiation within a solid angle of $\pm 1.2^{\circ}$ is refocused onto the entrance slit of an EUV flat field spectrometer, and the spectrally resolved photon signal is recorded by a phosphor screen microchannel plate (MCP) assembly and a charge coupled device (CCD) camera. Accurate spectrometer calibration was verified with plasma line emission and conventional high harmonic generation using amplified pulses.
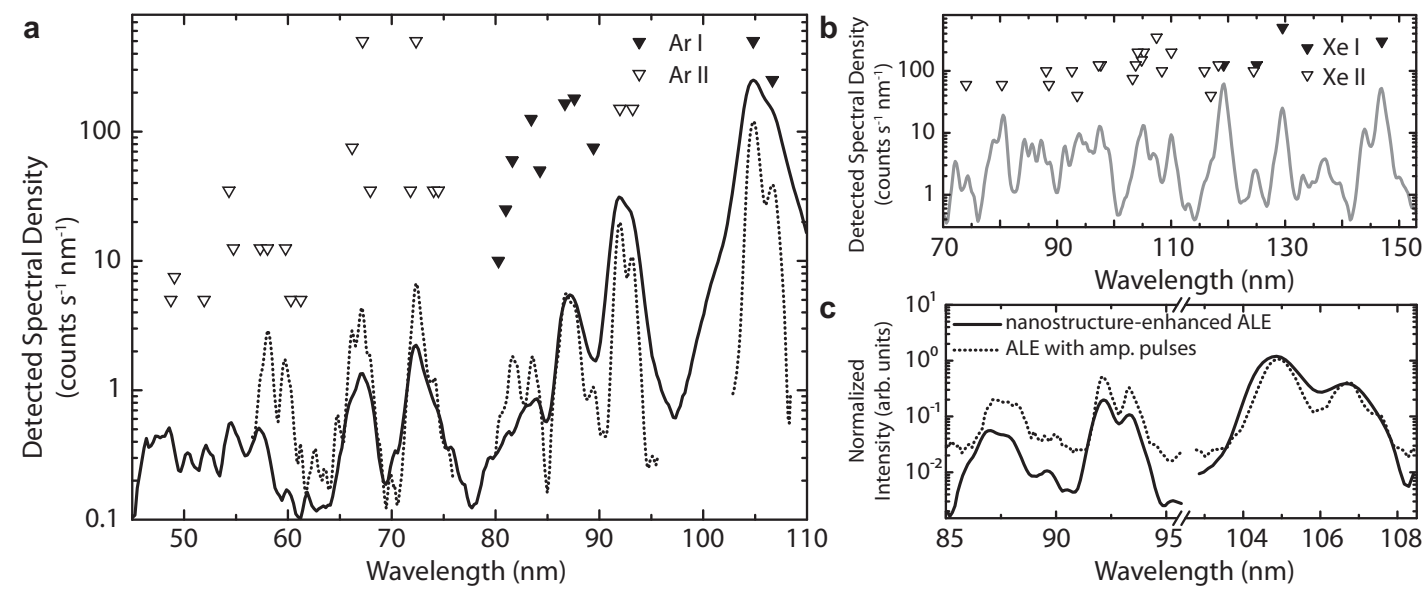

Fig. 2. Detected spectral density of nanostructure-enhanced ALE from argon (a) and xenon (b) gas. Solid and doted lines represent the first and second grating di ffraction orders, respectively. The expected wavelength positions of atomic transitions are indicated with filled (neutral gas) and open (singly ionized gas) triangles, and the triangle heights correspond to the relative intensities documented for plasma discharge [7]. c) Comparison of the nanostructure-enhanced ALE spectrum and an ALE spectrum taken with amplified mJ-pulses from a $1 \mathrm{kHz}$ laser system. The incident intensities are $0.15 \mathrm{TWcm}^{-2}$ and $98 \mathrm{TWcm}^{-2}$ for the ALE with nanostructures and amplified pulses, respectively.

\section{Results and discussion}

Figure 2 shows the spectrally resolved EUV emission from argon (a) and xenon (b) gas using nanostructures (cf. Fig. 1b) for local field-enhancement. The detected peaks, with maximum count rates exceeding one hundred detected photons per second and nanometer spectral bandwidth, correspond to multiple emission lines. The origin of this emission is atomic fluorescence, as indicated with the expected positions of atomic line transitions for neutral (solid triangles) and singly ionized (open triangles) atoms of the respective gas species. Triangle heights represent the documented relative intensities [7]. Some additional lines in the xenon spectrum most likely correspond to emission from higher ionized atoms and diffraction from higher orders. 
In order to gauge the locally enhanced intensities achieved in the bow ties, we have carried out reference measurements in the absence of nanostructures, using amplified pulses to excite argon fluorescence at variable intensities exceeding $10^{14} \mathrm{Wcm}^{-2}$ (cf. Fig. 1d). Comparing the intensity dependent spectral characteristics (e.g. variations in relative line intensities) of the reference measurements with the nanostructure-enhanced ALE spectrum (cf. Fig. 2c), one can obtain an estimate for the local peak intensity in the nanostructure gap. This leads to a field-enhancement factor as high as $\sim 25$ relative to the incident field.

In our experiments, despite local intensities of about $100 \mathrm{TWcm}^{-2}$ and substantial ionization rates, we could not confirm any signature of the nanostructure-enhanced HHG previously reported [5]. We believe that HHG is much less efficiently enhanced in geometries using nanostructures for field-enhancement by confining the field region to very small (nanometric) volumes. This drastically reduces the coherent emission volume and thus the achieved conversion efficiencies [6]. In contrast, incoherent emission is less affected by these considerations, scaling linearly with the number of radiating dipoles. Hence, as this study demonstrates, plasmonic nanostructures can be utilized for efficient enhancement of incoherent multiphoton and strong-field excited atomic line emission.

The present experiments and considerations illustrate fundamental limitations to nanostructureenhanced HHG in optical near-fields and suggest that alternative approaches may have to taken.

\section{References}

1. A. Bouhelier, M. Beversluis, A. Hartschuh, L. Novotny, Phys. Rev. Lett. 90, 013903 (2003)

2. H. Fischer, O. J. F. Martin, Opt. Express 16, 9144 (2008)

3. T. Hanke, G. Krauss, D Träutlein, B. Wild, R. Bratschitsch, A. Leitenstorfer, Phys. Rev. Lett. 103, 257404 (2009)

4. G. Herink, M. Gulde, D. R. Solli, C. Ropers, Nature 483, 190 (2012)

5. S. Kim, J. Jin, Y.-J. Kim, I.-Y. Park, Y. Kim, S.-W. Kim, Nature 453, 757 (2008)

6. M. Sivis, M. Duwe, B. Abel, C. Ropers, Nature 485, E1 (2012)

7. J. E. Sansonetti, W. C. Martin, J. Phys. Chem. Ref. Data 34, 1559 (2005) 